\title{
Basi-parallel Anatomical Scanning Magnetic Resonance Imaging in Patients With Bilateral Vertebrobasilar Artery Dissections -Case Report-
}

\author{
Junkoh YAMAмOTO, ${ }^{1}$ Naoto SAKAI, ${ }^{1}$ and Tetsuo YOKOYAMA ${ }^{1}$ \\ ${ }^{1}$ Department of Neurosurgery, Omaezaki Municipal Hospital, Omaezaki, Shizuoka
}

\begin{abstract}
A 31-year-old man presented with bilateral unruptured vertebral artery (VA) dissections. The temporal course of the dissected arteries was successively evaluated using brain surface imaging modality basiparallel anatomical scanning (BPAS) magnetic resonance (MR) imaging in combination with the conventional modalities of MR angiography and three-dimensional computed tomography (3D-CT) angiography. Initially, BPAS-MR imaging clearly demonstrated bilateral fusiform dilatations of the arterial wall, whereas MR angiography and 3D-CT angiography showed irregular, dilated, or interrupted inner contour of the arteries. Finally, BPAS-MR imaging demonstrated resolution of both aneurysms, and MR angiography demonstrated obstruction of the left VA and normal contours of the right VA. Combination of diagnostic tools such as BPAS-MR imaging and MR angiography is useful for evaluating the exact nature of dissected arteries and determining the temporal course.
\end{abstract}

Key words: vertebral artery, magnetic resonance imaging, fusiform aneurysm, brain stem infarction, magnetic resonance cisternography

Received September 6, 2010; Accepted January 6, 2011 


\section{Introduction}

Spontaneous dissection of the vertebrobasilar artery (VA) may develop into subarachnoidal hemorrhage (SAH) or brain ischemia. ${ }^{17,21)}$ The incidence of rebleeding and the mortality rate are higher in patients who developed $\mathrm{SAH}$ compared to patients with unruptured VA dissection,7) whereas favorable outcomes occur in patients with unruptured VA. ${ }^{3,16,22)}$ However, the pathogenesis and exact natural history of VA dissection remain unclear. Basiparallel anatomical scanning (BPAS) magnetic resonance (MR) imaging is a simple MR imaging technique that modifies surface anatomical scanning and can depict the surface appearance of the intracranial VA. ${ }^{4,12,13)}$

We describe a case of bilateral VA dissections in which the temporal course of the dissected arteries was serially evaluated using BPAS-MR imaging in addition to MR angiography and three-dimensional computed tomography (3D-CT) angiography, and discuss the use of BPAS-MR imaging in conjunction with conventional imaging tools to achieve accurate diagnosis and evaluation of the temporal course of the disease in patients with VA dissection.

\section{Case Report}

A 31-year-old man noticed slight dull sensation in the suboccipital region and complained of nausea one week before admission to our hospital. On admission, he complained of persistent dull sensation and stiffness in the
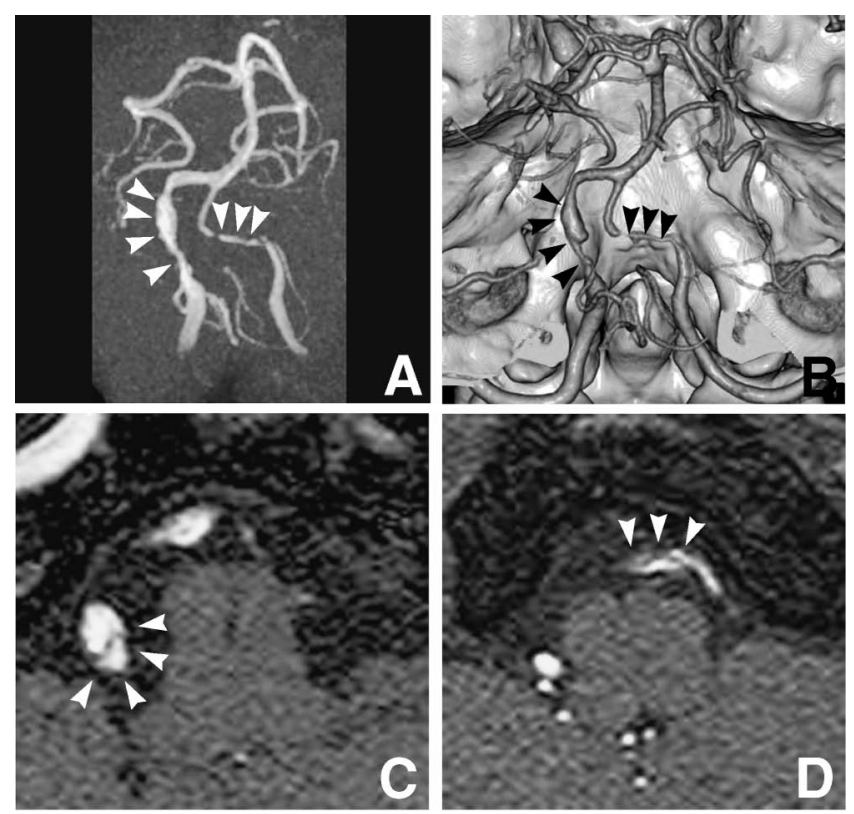

Fig. 1 Studies obtained on admission. A: Magnetic resonance (MR) angiogram showing a fusiform dilatation of the artery with proximal narrowing of the left vertebral artery (VA), and tapered narrowing of the right VA (arrowheads). B: Three-dimensional computed tomography angiogram showing similar findings (arrowheads). C, D: MR angiograms showing double lumen signs in the left and right VAs (arrowheads). suboccipital and neck region without headache. Neurological examination revealed no deficits. The symptoms were suggestive of SAH, but CT and lumbar puncture, and conventional $M R$ imaging, including diffusion-weighted imaging, showed no abnormality. MR and 3D-CT angiography showed essentially the same findings of abnormal contours of the artery just distal to the posterior inferior cerebellar artery (PICA), elongated fusiform dilatation with proximal narrowing in the left $\mathrm{VA}$, and tapered narrowing in the right VA (Fig. 1A, B). MR angiography showed double lumen in the fusiform dilatation of the left VA (Fig. 1C) and in the right VA (Fig. 1D).

Under a diagnosis of unruptured bilateral VA dissections, we treated the patient conservatively with hydration
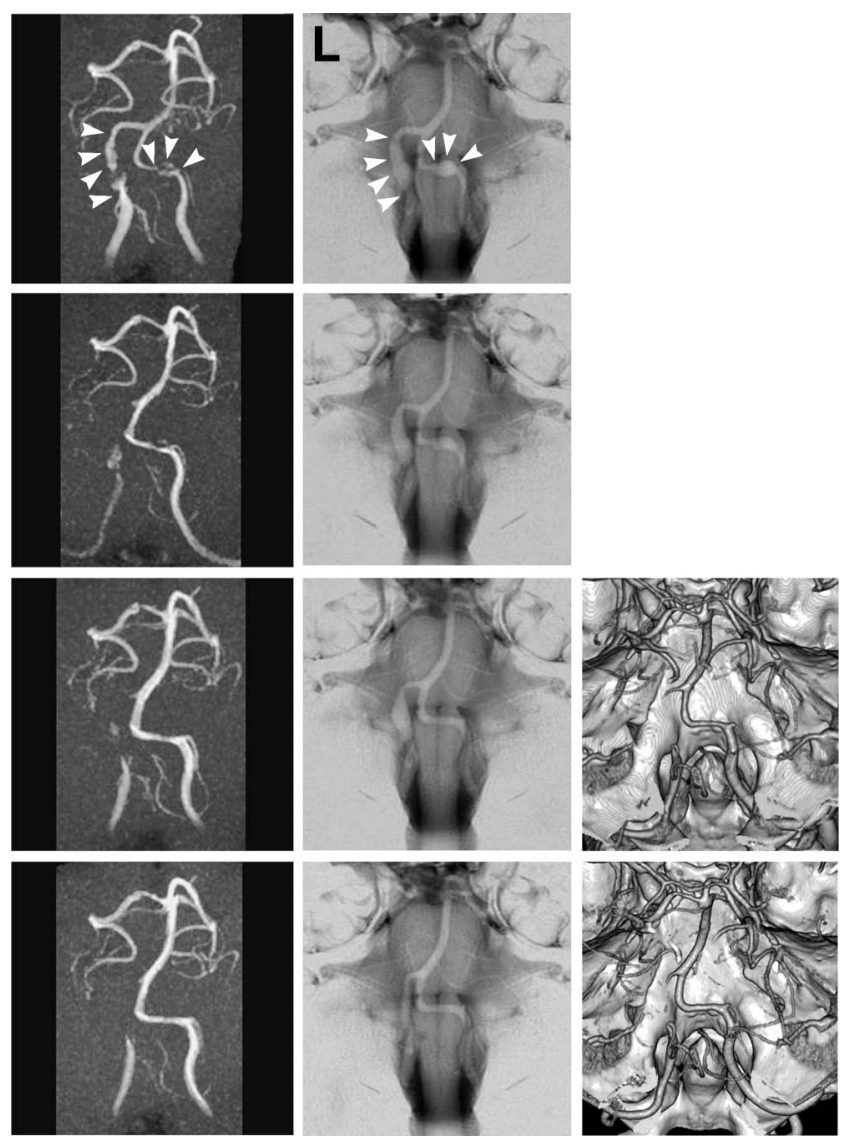

Fig. 2 Sequential follow-up studies using magnetic resonance (MR) angiography (left column), basi-parallel anatomical scanning (BPAS)-MR imaging (center column), and three-dimensional computed tomography (3D-CT) angiography (right column) at 2, 4, 7, and 11 weeks (upper row, upper middle row, lower middle row, and lower row, respectively). Fusiform dilatation of the arterial wall was clearly visible on BPAS-MR images and MR angiograms, with irregular, dilated, or interrupted inner contour of the artery on 3D-CT angiograms at 2 weeks. The disrupted inner contour of the right vertebral artery (VA) returned to normal at 7 weeks, whereas that of the left VA extended to the VA-basilar artery junction at 4 weeks and was completely obstructed at 11 weeks. The right fusiform aneurysm resolved at 7 weeks, and the left fusiform aneurysm at 11 weeks on BPAS-MR images. 
and medication to prevent cerebral ischemia or SAH. Two weeks after the onset, MR angiography showed an interrupted and irregular lumen within the elongated fusiform dilatation in the left VA, in contrast to focal dissection maintaining vessel patency in the right VA (Fig. 2). BPASMR imaging using parameters of slice thickness, $25 \mathrm{~mm}$; time to repetition/echo, 5000/763 msec; field of view, 160 $\times 160 \mathrm{~mm}$; matrix, $384 \times 224$; and acquisition time, 25 sec showed an elongated fusiform dilatation and a small aneurysm in the left and right VAs, respectively. Thereafter, the patient's condition suddenly deteriorated. He developed dysphagia, hoarseness, and dizziness 4 weeks after onset. MR angiography showed proximal dilatation of the lesion in the left VA, and disappearance of the flow signal that extended to the VA-basilar artery (BA) junction (Fig. 2). MR angiography of the right VA showed that the inner contour of the artery had begun to regain its normal appearance, although a part of the vessel was still disrupted (Fig. 2). BPAS-MR imaging 4 weeks after onset showed that the appearance of the left VA remained essentially the same as at 2 weeks after onset, but the right VA showed shrinkage of the fusiform dilatation (Fig. 2). Conventional MR imaging including diffusion-weighted imaging showed no abnormality within the brain stem, but we treated the patient with antiplatelet medication and edaravone, a free radical scavenger, ${ }^{19,20)}$ under a diagnosis of brain stem ischemia due to interrupted blood flow in the left VA.

Seven weeks after onset, MR and 3D-CT angiography showed residual fusiform dilatation of the left VA and occlusion of the true lumen just distal to the PICA. However, the inner contour of the right VA had regained its normal appearance (Fig. 2). Seven weeks after onset, BPAS-MR imaging showed a slight increase of the mid portion and tapering of the distal portion in the fusiform dilatation in the left VA, and normal appearance of the right VA (Fig. 2). Eleven weeks after onset, the patient's neurological deficits had markedly improved, so that he was able to carry on his daily activities without difficulty. Eleven weeks after the onset, MR and 3D-CT angiography showed occlusion of the left VA from the VA-PICA junction to the VABA junction (Fig. 2). BPAS-MR imaging showed that the fusiform dilatation of the left VA had disappeared and the artery had regained its normal appearance. Two months after onset, the patient was discharged and returned to his previous occupation.

\section{Discussion}

The prognosis for patients with unruptured VA dissection is generally better than in patients with ruptured VA dissection. Unruptured VA dissection often improves spontaneously, with a high reported incidence (62\%) of spontaneous angiographic cure. ${ }^{22)}$ However, subintimal dissection extending into the BA, or persistent aneurysmal dilatation occurring as a sequel to artery dissection, are associated with brain stem infarction or delayed SAH.1,8) Therefore, serial vascular evaluation is very important to monitor the time course of changes in patients with unruptured VA dissection.
The present relatively rare case of bilateral VA dissections exhibited different temporal courses of the disease in the right and left VAs. The dissection was short in the right VA, and the configuration of the true lumen was relatively smooth, so maintaining blood patency. In contrast, the dissection was extended in the left VA with severe irregularities of the true lumen. Consequently, the dissected arterial wall of the right VA improved spontaneously and the arterial caliber normalized, whereas the left VA lumen remained obstructed. A case of rupture of a VA dissecting aneurysm immediately after trapping of a dissecting aneurysm on the contralateral VA was reported. ${ }^{6)}$ In our case, initial MR angiography showed the dominant blood flow was in the left VA. In addition, stenosis of the right VA may increase blood flow, and so induce hemodynamic stress in the left VA. These phenomena may also cause severe irregularity of the lumen in the left VA. Therefore, we conclude that the length of the dissected vessel, the luminal configuration, and the dominant blood flow in the VA are the main factors which influence the natural course of unruptured VA dissection.

Conventional angiography is still considered the gold standard in the diagnosis of VA dissection, but has been gradually replaced by MR imaging, MR angiography, and CT angiography. $\mathrm{T}_{1}$-weighted $\mathrm{MR}$ imaging shows intramural hematoma, which characteristically appears as a crescent-shaped area around the eccentric true lumen. ${ }^{14)}$ However, $\mathrm{T}_{1}$-weighted MR imaging may also show flowrelated enhancement of the vertebral venous plexus, thus mimicking the appearance of intramural hematoma. ${ }^{10)} 3 \mathrm{D}$ time of flight (TOF) MR angiography may reveal what appears to be an increased external diameter of the artery due to the superimposed intramural hematoma containing methemoglobin. This finding is very useful for the detection of carotid artery dissection, but is of limited use for VA dissection because of the small diameter and marked variation in arterial caliber. ${ }^{9)}$ The sensitivities of MR imaging and 3D-TOF MR angiography for the detection of VA dissection are $20-60 \%$ and $60 \%$, respectively. ${ }^{9,23)}$ In contrast, multidetector 3D-CT angiography has a high accuracy for the diagnosis of VA dissection, with sensitivity and specificity of $100 \%$ and $98 \%$, respectively. However, there are some complicating factors such as severely atherosclerotic vessels with calcification, radiation exposure, and administration of contrast reagents. ${ }^{2)}$ If the vessel dissection enlarges distally, the MR signals in the pseudo-lumen of dissected vessels are affected by the turbulence of blood flow, and 3D-TOF MR angiography may not clearly depict blood flow within the pseudo-lumen. ${ }^{5,11,15)}$ Thus, MR angiography may underestimate the area of dissection. Moreover, MR angiography evaluation of the absence or decrease in the blood flow signal may not clearly discriminate atheromatous changes from resolving vascular dissections. ${ }^{12)}$ Therefore, we consider that evaluation of the dissection of vessels should include examination of both the inner and outer contours of the vessels.

BPAS-MR imaging reflects the outer surface appearance of the intracranial arterial wall, whereas MR and 3DCT angiography mainly reflect the blood flow within the artery, so representing the inner contour of the artery. 
Moreover, BPAS-MR imaging does not require a long sequence time compared to 3D-TOF MR angiography with contrast medium. Therefore, BPAS-MR imaging of the artery in conjunction with conventional MR angiography or 3D-CT angiography will enable precise evaluation of both the inner and outer contours of the artery, so establishing accurate diagnosis of arterial wall lesions, such as VA dissection or atheromatous changes in the artery. ${ }^{12,13,18)}$

In our case, BPAS-MR imaging clearly demonstrated a fusiform dilatation of the arterial wall, whereas MR and 3D-CT angiography showed irregular, dilated, or disrupted inner contour of the artery. In the left VA, MR angiography revealed persistent aneurysmal dissection (fusiform dilatation) and progressive stenosis (occlusion) extending to the VA-BA junction from 2-7 weeks. In contrast, BPAS-MR imaging did not reveal any extension of the VA dissection, only slight dilation of the mid portion of the fusiform aneurysm with gradual tapering of the distal portion. We hypothesize that dilation of the mid portion in the fusiform aneurysm by partial thrombosis of the pseudo-lumen induced stenosis of the true lumen, resulting in decreased blood flow, and tapering of the distal portion of the aneurysm. Consequently, the pseudo-lumen was completely occluded, and the VA dissection subsided. These morphological changes of a fusiform dilatation observed by BPAS-MR imaging might indicate a favorable course in an unruptured dissected VA aneurysm. Therefore, we suggest that sequential follow-up study of the dissected fusiform aneurysm using both conventional MR angiography and BPAS-MR imaging is very important to predict the prognosis.

\section{References}

1) Caplan LR, Baquis GD, Pessin MS, D'Alton J, Adelman LS, DeWitt LD, Ho K, Izukawa D, Kwan ES: Dissection of the intracranial vertebral artery. Neurology 38: 868-877, 1988

2) Chen CJ, Tseng YC, Lee TH, Hsu HL, See LC: Multisection CT angiography compared with catheter angiography in diagnosing vertebral artery dissection. AJNR Am J Neuroradiol 25: 769-774, 2004

3) Fukunaga A, Tabuse $M$, Naritaka $H$, Nakamura $T$, Akiyama T: Spontaneous resolution of nontraumatic bilateral intracranial vertebral artery dissections. Neurol Med Chir (Tokyo) 42: 491-495, 2002

4) Hamaguchi T, Yamada M: Basiparallel anatomic scanningmagnetic resonance imaging in vertebral artery dissection. Arch Neurol 66: 276-277, 2009

5) Jou LD, Wong G, Dispensa B, Lawton MT, Higashida RT, Young WL, Saloner D: Correlation between lumenal geometry changes and hemodynamics in fusiform intracranial aneurysms. AJNR Am J Neuroradiol 26: 2357-2363, 2005

6) Katsuno M, Mizunari T, Kobayashi S, Takahashi H, Teramoto A: Rupture of a vertebral artery dissecting aneurysm developing immediately after trapping of a dissecting aneurysm on the contralateral vertebral artery: case report. Neurol Med Chir (Tokyo) 49: 468-470, 2009

7) Kim CH, Son YJ, Paek SH, Han MH, Kim JE, Chung YS, Kwon BJ, Oh CW, Han DH: Clinical analysis of vertebrobasilar dissection. Acta Neurochir (Wien) 148: 395-404, 2006

8) Kitanaka C, Tanaka J, Kuwahara M, Teraoka A, Sasaki T, Takakura K, Tanaki J: Nonsurgical treatment of unruptured intracranial vertebral artery dissection with serial follow-up angiography. J Neurosurg 80: 667-674, 1994

9) Levy C, Laissy JP, Raveau V, Amarenco P, Servois V, Bousser MG, Tubiana JM: Carotid and vertebral artery dissections: three-dimensional time-of-flight MR angiography and MR imaging versus conventional angiography. Radiology 190: 97-103, 1994

10) Miaux Y, Cognard C, Martin-Duverneuil N, Weill A, Savin D, Chiras J: Flow-related enhancement in the vertebral plexus mimicking an intramural hematoma. AJNR Am J Neuroradiol 17: 191-192, 1996

11) Mustert BR, Williams DM, Prince MR: In vitro model of arterial stenosis: correlation of MR signal dephasing and trans-stenotic pressure gradients. Magn Reson Imaging 16: 301-310, 1998

12) Nagahata M, Abe $Y$, Ono S, Hosoya T, Uno S: Surface appearance of the vertebrobasilar artery revealed on basiparallel anatomic scanning (BPAS)-MR imaging: its role for brain MR examination. AJNR Am J Neuroradiol 26: 2508-2513, 2005

13) Nagahata M, Manabe H, Hasegawa S, Takemura A: Morphological change of unruptured vertebral artery dissection on serial MR examinations. Evaluation of the arterial outer contour by basi-parallel anatomical scanning (BPAS)-MRI. Interv Neuroradiol 12: 133-136, 2006

14) Nagahiro S, Hamada J, Sakamoto Y, Ushio Y: Follow-up evaluation of dissecting aneurysms of the vertebrobasilar circulation by using gadolinium-enhanced magnetic resonance imaging. J Neurosurg 87: 385-390, 1997

15) Nederkoorn PJ, van der Graaf $Y$, Eikelboom BC, van der Lugt A, Bartels LW, Mali WP: Time-of-flight MR angiography of carotid artery stenosis: does a flow void represent severe stenosis? AJNR Am J Neuroradiol 23: 1779-1784, 2002

16) Pozzati E, Padovani R, Fabrizi A, Sabattini L, Gaist G: Benign arterial dissections of the posterior circulation. J Neurosurg 75: 69-72, 1991

17) Savitz SI, Caplan LR: Vertebrobasilar disease. N Engl J Med 352: 2618-2626, 2005

18) Takada H, Hyogo T, Kataoka T, Hayase K, Nakamura H: Diagnosis of vertebral artery dissection by basi-parallel anatomical scanning (BPAS) MRI. Interv Neuroradiol 12: 129-132, 2006

19) Tanaka M: [Pharmacological and clinical profile of the free radical scavenger edaravone as a neuroprotective agent]. Nippon Yakurigaku Zasshi 119: 301-308, 2002 (Japanese)

20) Yamamoto J, Nishizawa $S$, Yamaguchi M, Akamine $S$, Tokuyama T, Sugiyama $K$, Namba $H$ : Differences in diffusion-weighted and T2-weighted magnetic resonance imaging findings in the acute and chronic stages of ischemic cerebrovascular disease- two case reports. Neurol Med Chir (Tokyo) 44: 245-248, 2004

21) Yamaura A, Ono J, Hirai S: Clinical picture of intracranial non-traumatic dissecting aneurysm. Neuropathology 20: 85-90, 2000

22) Yoshimoto Y, Wakai S: Unruptured intracranial vertebral artery dissection. Clinical course and serial radiographic imagings. Stroke 28: 370-374, 1997

23) Zuber M, Meary E, Meder JF, Mas JL: Magnetic resonance imaging and dynamic CT scan in cervical artery dissections. Stroke 25: 576-581, 1994

Address reprint requests to: Junkoh Yamamoto, MD, PhD, Department of Neurosurgery, University of Occupational and Environmental Health, 1-1 Iseigaoka, Yahata-Nishi, Kitakyushu, Fukuoka, 807-8555, Japan. e-mail: yama9218@med.uoeh-u.ac.jp 